\title{
Controlling the spatial distribution of quantum dots in nanofiber for light-harvesting devices
}

\author{
Yung Ji Choi ${ }^{1}$, Daesub Hwang ${ }^{1,2}$, Heejae Chung ${ }^{1}$, Dong Young Kim $^{2}$ and Dongho Kim ${ }^{1}$ \\ The ability to control inter-dot or inter-molecule spacing of functional moieties in solid-state devices has long been studied for \\ both fundamental and technological reasons. In this study, we present a new strategy for controlling the distance between \\ quantum dots (QDs) based on one-dimensional spatial confinement in a polymer nanofiber template. This reliable technique \\ allows for the isolation of QDs at a sufficient distance in a thin film and retains their monomeric character, with distinct spectra \\ from aggregates ( 30-nm shift) and monoexponential photoluminescence decay, indicating the suppression of inter-dot \\ interactions. We successfully developed light-harvesting devices by incorporating QDs in nanofibers as an auxiliary light \\ harvester, improving the performance of these devices from 5.9 to $7.4 \%$. This strategy offers a viable path of controlling the \\ arrangements of various functional moieties in solid-state devices.
}

NPG Asia Materials (2015) 7, e202; doi:10.1038/am.2015.76; published online 17 July 2015

\section{INTRODUCTION}

The physicochemical properties of functional moieties in devices such as organic dyes, quantum dots (QDs), graphenes and other nanostructures are different from the properties of their aggregates, ${ }^{1-7}$ which frequently deteriorate the performance of the optoelectronic devices (light-emitting diodes, lasers, solar cells, waveguides or photosensors) ${ }^{8-14}$ For instance, QD light-emitting diodes or photovoltaic devices suffer from the inter-dot interactions of aggregates, which cause a spectral shift and a broadening in the photoluminescence of QDs as well as a decrease in the quantum yield. ${ }^{10,13}$ Therefore, control of inter-dot spacing is critical in ensuring device performance. ${ }^{8-10,13,15}$ Researchers have long sought methods to control the spatial distribution of functional moieties in devices and prevent the formation of aggregates, both for fundamental research and the potential technological applications. In solid-state films in particular, it has been challenging to increase film thickness while keeping functional moieties in the monomeric state.

Here, we demonstrate a general strategy for controlling the distance between functional moieties based on one-dimensional spatial confinement in a polymeric nanofiber template. One key observation, shown both experimentally and computationally, is that as the radius of a nanofiber template becomes smaller, the distance between moieties increases, thus leading to substantial isolation of each dot. QDs were chosen as a representative functional moiety because they can be easily observed by electron microscopy and because they have a high quantum yield, excellent photostability and long intrinsic lifetime that is distinguishable from their inter-dot energy transfer time (50 ps$2 \mathrm{~ns}) .{ }^{15,16}$ We have fabricated isolated QDs (iQDs) by confining QDs in a narrow polymer nanofiber, and the resulting structure was studied with a confocal luminescence microscope, transmission electron microscope and scanning electron microscope. By systematically elucidating the optical characteristics of this material, such as its photoluminescence spectrum and its decay profile, we discovered that iQDs have distinct photoluminescence spectra and lifetimes compared with typical two-dimensional QD aggregates. These results represent the monomeric properties of iQDs in solid-state nanofiber films. Moreover, we evaluated the potential of using spatial control technique in various devices and, as a brief example, tested the applicability of $\mathrm{QQD}$ as an auxiliary light harvester to improve the performance of Förster resonant energy transfer (FRET)-based lightharvesting devices (over 25\%).

\section{MATERIALS AND METHODS}

Fabrication of fiber-embedded iQDs

First, 1.0-2.0 g poly(methyl methacrylate) (PMMA; Mn $=990000,99 \%$ hydrolyzed) was dispersed in $10 \mathrm{ml}$ of chloroform with vigorous stirring for $\sim 2 \mathrm{~h}$, followed by the addition of $1 \mathrm{ml}$ of CdSe solution (toluene). The resulting homogeneous solution $(0.24-1$ or $1-5 \mathrm{wt} \%$ CdSe for the single-molecule spectroscopic study or the ensemble spectroscopic study, respectively) was used for electrospinning. A needle with a $\mathrm{Cu}$ rod inserted to connect the highvoltage supply was filled with the CdSe/chloroform solution. An Al sheet connected to the ground was used as the receiver. The distance between the burette tip and receiver was fixed at $10 \mathrm{~cm}$, and the high-voltage supply was fixed at $13 \mathrm{kV}$. The spinning rate was controlled at $\sim 20 \mu \mathrm{m}^{-1}$ using a syringe infusion pump (KDS200, KD Scientific, Holliston, MA, USA). The films were cast by electrospinning the composite solution onto a 1.5 -mm-thick quartz

${ }^{1}$ Spectroscopy Laboratory for Functional $\pi$-electronic Systems, Department of Chemistry, Yonsei University, Seoul, Korea and ${ }^{2}$ Optoelectronic Materials Lab, Korea Institute of Science and Technology, Seoul, Korea

Correspondence: Professor D Kim, Spectroscopy Laboratory for Functional $\pi$-electronic Systems, Department of Chemistry, Yonsei University, 134 Shinchon-dong, Seodaemungu, Seoul 120-749, Korea.

E-mail: dongho@yonsei.ac.kr

Received 2 January 2015; revised 22 May 2015; accepted 25 May 2015 
crystal plate or thoroughly cleaned microscope cover slips to ensemble spectroscopy or single-molecule spectroscopy, respectively.

\section{Device fabrication}

To prepare the working electrode of the dye-sensitized solar cell, a fluorinedoped $\mathrm{SnO}_{2}$ (FTO)-conducting glass substrate (TEC 8, Pilkington, Tokyo, Japan) was first washed with a detergent solution in a sonicator bath for $10 \mathrm{~min}$ and then rinsed with deionized water and ethanol. The clean FTO glass substrates were then coated with the hierarchically structured nanobeads composed of anatase $\mathrm{TiO}_{2}$ nanoparticles and nanofibers enveloping CdSe QD obtained via the simultaneous electrospray and electrospinning method. To obtain a uniform thickness over a large area, the nozzle and the substrate were placed on a motion control system regulated by a microprocessor. The thickness of the active layer was measured and found to be 7-8 $\mu \mathrm{m}$ using an Alpha-step 200 surface profiler (Tencor, Milpitas, CA, USA). The working electrode was immersed into $0.1 \mathrm{M}$ aqueous $\mathrm{TiCl}_{4}$, followed by washing with deionized water and ethanol, and then sintered at $200^{\circ} \mathrm{C}$ for $30 \mathrm{~min}$. After cooling to $80^{\circ} \mathrm{C}$, the working electrodes were immersed in solutions of $\beta$ - $(4-$ ethynylbenzoic acid)-substituted zinc porphyrin (ZnEP1, $0.2 \mathrm{~mm}$ in ethanol) and allowed to stand overnight (15-20 h).

To prepare the counter electrode, the FTO plates were drilled and washed first with deionized water and then $0.1 \mathrm{M}$ hydrochloric acid in ethanol with sonication for $10 \mathrm{~min}$. After the FTO glass was washed, the counter electrodes were prepared by drop casting a solution of $\mathrm{H}_{2} \mathrm{PtCl}_{6}(0.5 \mathrm{~mm}$ in isopropyl alcohol) onto the FTO glass and then heating at $400^{\circ} \mathrm{C}$ for $20 \mathrm{~min}$. The dyeadsorbed $\mathrm{TiO}_{2}$ films and the platinum counter electrodes were assembled into sealed sandwich-type cells by heating with a hot-melt film (Surlyn 1702, $25 \mu \mathrm{m}$ thickness, Dupont, Wilmington, DE, USA) used as a spacer between the electrodes. An aliquot of the electrolyte solution $\left(0.22 \mathrm{M}\left[\mathrm{Co}(\text { bpy })_{2}\right]^{2+}, 0.05 \mathrm{M}\right.$ $\left[\mathrm{Co}(\text { bpy })_{3}\right]^{3+}, 0.1 \mathrm{M} \mathrm{LiClO}_{4}$ and $0.5 \mathrm{M}$ 4-tert-butylpyridine in acetonitrile) was then placed into a hole in the counter electrode of the assembled cells. The hole was sealed with a microscope cover glass and surlyn $(25 \mu \mathrm{m})$ to prevent leakage of the electrolyte solution. The experimental details of the measurement of photovoltaic properties are described in Supplementary Note 1.

\section{Ensemble spectroscopic measurement}

Steady-state absorption spectra in solution and on transparent $\mathrm{TiO}_{2}$ were acquired using an Ultraviolet-visible-near infrared spectrometer (Varian, Cary5000, Agilent Technologies, Santa Clara, CA, USA). Steady-state photoluminescence spectra were recorded on a photoluminescence spectrometer (FluoroMate FS-2, SCINCO, Seoul, Korea). Time-resolved photoluminescence was detected using a time-correlated single-photon counting (TCSPC) technique to measure spontaneous photoluminescence decay. The excitation light source was a mode-locked Ti:sapphire laser (MaiTai BB, Spectra-Physics, Santa Clara, CA, USA), which provides ultrashort pulses ( $80 \mathrm{fs}$ at full-width halfmaximum) with a high repetition rate $(80 \mathrm{MHz})$. This high repetition rate may be slowed to $1 \mathrm{MHz}-800 \mathrm{kHz}$ using a homemade pulse picker. The pulsepicked output pulse was frequency-doubled by a 1-mm-thick BBO crystal (EKSMA, Vilnius, Lithuania). The photoluminescence was collected by a microchannel plate photomultiplier (R3809U-51, Hamamatsu, Hamamatsu, Japan) with a thermoelectric cooler (C4878, Hamamatsu) connected to a TCSPC board (SPC-130, Becker\&Hickl GmbH (Berlin, Germany)). The overall instrumental response function was $\sim 25 \mathrm{ps}$ (full-width half-maximum). A pump pulse vertically polarized by a Glan-laser polarizer was used to irradiate the samples, and a sheet polarizer, set at an angle complementary to the magic angle $\left(54.7^{\circ}\right)$, was placed in the photoluminescence collection path to obtain polarization-independent photoluminescence decays.

\section{Single-molecule spectroscopic measurement}

Single-molecule photoluminescence was detected with a confocal microscope (TE2000-U, Nikon) equipped with a sample scanning stage (XE-120, Park Systems, Suwon, Korea) at room temperature under ambient conditions. The sample was excited by circularly polarized light from a picosecond-pulsed diode laser (LDH-F-C-470, Picoquant (Berlin, Germany), $470 \mathrm{~nm}, 10 \mathrm{MHz}$ repetition rate, 70 ps pulse width) from a Berek compensator (5540, New Focus, Santa Clara, CA, USA). The laser beam was directed into the microscope by passing through a laser line filter (z470/10x, Chroma Technology, Bellows Falls, VT, USA) and collimating lens and was focused onto the sample via an oil immersion objective lens (Plan Fluor, 1.3 NA, $100 \times$, Nikon, Tokyo, Japan), yielding an irradiation power of $200 \mathrm{~W} \mathrm{~cm}^{-2}$ at the sample. Photoluminescence was collected using the same objective, passed through a dichroic mirror (470dcxru, Chroma Technology), filtered with a notch filter (HNPF-470.0-1.0, Kaiser Optical Systems, Ann Arbor, MI, USA) as well as band-pass filters (HQ488 lp and ET542 lp, Chroma Technology), and focused on an avalanche photodiode (SPCM-AQR-16-FC, PerkinElmer, Waltham, MA, USA). After an individual molecule was positioned in the focus of the laser, the photoluminescence signal detected by the avalanche photodiode was registered by a TCSPC PC card (SPC 830, Becker \& Hickl) operated in the first-in-first-out regime, in which the arrival time after the beginning of the acquisition and the time lag with respect to the excitation pulse were stored for each detected photon. The full-width half-maximum of the overall instrumental response function was $\sim 500-600 \mathrm{ps}$. These data were processed using BIFL data analyzer software (Scientific Software Technologies Center, Minsk, Belarus) to obtain the fluorescence intensity trajectory (FITs) with a user-defined binning time, and the time-resolved photoluminescence decayed using photons belonging to a user-defined region in the trajectories.

Separated iQDs were imaged using a wide-field photoluminescence microscope consisting of an inverted optical microscope (IX71, Olympus, Tokyo, Japan) equipped with an oil immersion objective $(1.4 \mathrm{NA}, \times 100$, Plan Fluorite, Olympus) and a highly sensitive, cooled, $512 \times 512$ pixels EMCCD camera (iXon Ultra, Andor Technology, Belfast, UK). The samples were excited with $445 \mathrm{~nm}$ light from a CW Cube laser (Coherent, Cube, Santa Clara, CA, USA). The collimated, circularly polarized laser beam was sent to the epiphotoluminescence microscope after it was passed through a laser line filter (FF01-445/20-25, Semrock, Lake Forest, IL, USA), collimating lens and a dichroic mirror (Di02-R442, Chroma Technology). The image reached the charge-coupled device (CCD) camera after spectral filtering with a notch filter (HNPF-445.0AR-1.0, Kaiser optical system) and a band pass filter (BLP01458R, Semrock and ET542 lp, Chroma Technology). The integration time per frame was $0.3 \mathrm{~s}$

\section{RESULTS AND DISCUSSION}

A pristine CdSe quantum dot solution with trioctylphosphine as a capping ligand (Supplementary Note 1 and Supplementary Figure S1) and polymer composites was prepared. Well-distributed polymer composites of QDs were prepared by screening the solvent and polymer and manipulating the polymer concentration to prevent the aggregation of QDs. ${ }^{16}$ The QD solution was susceptible to aggregation in most solvents except for chloroform and acetone (Supplementary Figure S2) because most solvents change the overall polarity and break the subtle balance of the colloidal structures. PMMA was chosen as the fiber material due to its high solubility in chloroform, transparency and the ability to produce an extremely narrow diameter after electrospinning. ${ }^{17}$

Using the electrospinning process, the composite solution of QD (CdSe in toluene) and 10-19\% PMMA/chloroform was electrospun under an electric field of $1.3 \mathrm{kV} \mathrm{cm}^{-1}$ (Supplementary Figure S3). The sample solution was then flowed through a nozzle to which a high voltage was applied. The charged solution jet became sharply stretched and solidified during its path from the nozzle to the grounded metal plate, which served as a collector. Due to the elongational flow of the jet and the rapid solvent evaporation, QDs were expected to experience an extremely strong stretching force during the electrospinning process and to be arranged along the direction of elongation. ${ }^{18}$ Overall, the resulting QD nanofibers had fine structures with diameters of 25, 250, 500 and $1000 \mathrm{~nm}$. A Monte-Carlo simulation of distances between iQDs (Supplementary Note 2) predicted that fibers with diameters of $250 \mathrm{~nm}$ or less yield a sufficient distance ( $>1 \mu \mathrm{m}$ in $500 \mathrm{~nm}$ QD) between QDs. 
The scanning electron microscopy image shows the morphology of 250-nm-sized electrospun nanofibers enveloping iQDs, corresponding to the bright field confocal microscope image (Figures la and c). Furthermore, these nanofibers exhibited bright orange photoluminescence emission (Figure 1b) under ultraviolet light exposure at $363 \mathrm{~nm}$, with a color similar to that of the QD solution (inset of Figure 1b),
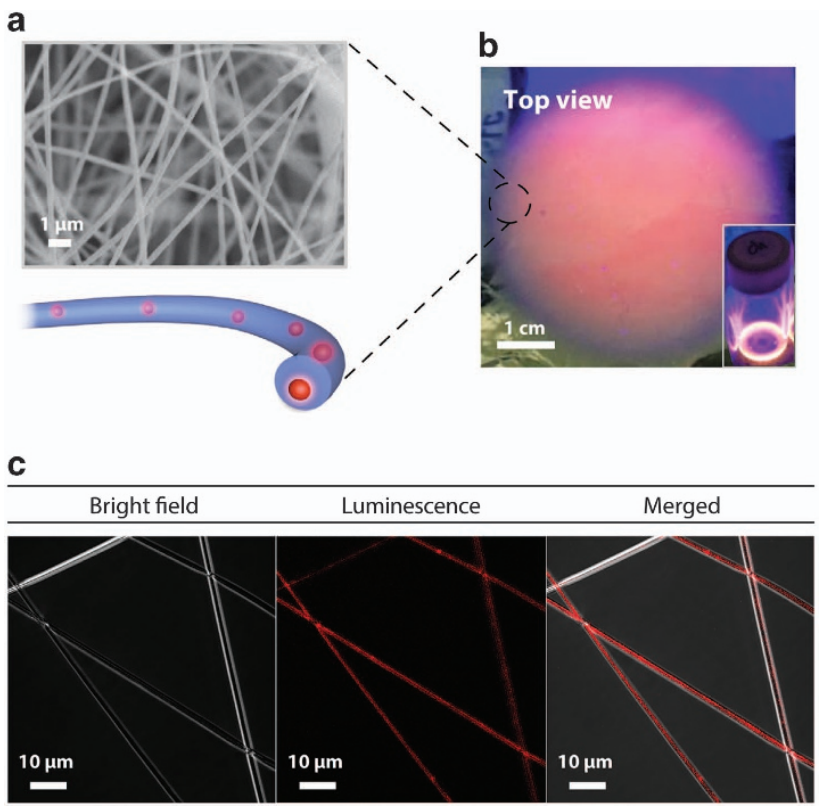

Figure 1 One-dimensional spatial confinement of quantum dots in the nanofiber. (a) Upper: scanning electron microscopy (SEM) image of electrospun nanofibers enveloping isolated quantum dots (iQDs). Lower: schematic figure of iQDs in the nanofiber. (b) Nanofibers enveloping iQDs were collected on aluminum foil and exhibited orange colored photoluminescence similar to the CdSe solution (inset) under ultraviolet light exposure $(363 \mathrm{~nm})$. (c) Bright field and luminescence images (left and middle, respectively) of nanofibers enveloping iQDs. Overlay of the bright field and luminescence images (right). while PMMA nanofibers without QDs did not exhibit this emission (Supplementary Figure S4). The photoluminescence of iQDs in the nanofiber was detected at $580 \mathrm{~nm}$ by confocal photoluminescence microscopy with photoexcitation at $532 \mathrm{~nm}$ (Figure 1c). Merging with the bright-field image shows that the photoluminescence signal was evenly distributed across the nanofiber, indicating that QDs are embedded along the nanofiber without forming aggregates, which would normally appear as bright spots (Supplementary Figure S5). To obtain direct information about the distribution of iQDs in the nanofiber, we captured transmission electron microscopy images of nanofiber samples (Figure 2a). In PMMA nanofibers with diameters of 25 and $250 \mathrm{~nm}$ (Figure 2a(i) and (ii), respectively), most iQDs with diameters of $5 \mathrm{~nm}$ were distributed without forming aggregates (inset of Figure 2a).

Thick nanofiber films, the thickness of which depends on the electrospinning time, were prepared to obtain suitable photoluminescence to ensemble spectroscopic measurements; an accumulation time of at least $10 \mathrm{~min}$ was required to obtain enough signal to perform ensemble measurements (500 nM QD). The steady-state photoluminescence spectrum of iQDs in the nanofiber (both 25 and $250 \mathrm{~nm}$ nanofibers) is similar to that of the solution, which was shifted to the shorter wavelength region compared with the two-dimensional QD aggregates (Figure $2 \mathrm{~b})$. A slight blue shift $(\sim 8 \mathrm{~nm})$ from the solution spectrum can be accounted for by the removal of the solvation effect. ${ }^{19}$ Moreover, the photoluminescence decay profiles of iQDs in the nanofiber, solution and two-dimensional QD aggregates (dropcast film) were obtained at 580, 590 and $610 \mathrm{~nm}$, respectively, by TCSPC measurements to reveal their exciton lifetimes. As seen in Figure $2 \mathrm{c}$, iQDs in the nanofiber exhibit nearly monoexponential decay ( $24 \mathrm{~ns})$ similar to that in solution; the photoluminescence decay curve of twodimensional QD aggregates, however, becomes multi-exponential with a large portion of rapid initial decay $(\sim 1 \mathrm{~ns})$. These results reflect that the exciton energy transfer due to dipole-dipole interactions affects the photoluminescence spectrum and its decay curve; ${ }^{4-7}$ the energy transfer from larger band-gap QDs to smaller band-gap QDs or defect states (Figure $2 \mathrm{~d}(\mathrm{i})$ ) results in a red-shifted emission peak ${ }^{4,5}$ and rapid photoluminescence decay $(50 \mathrm{ps}-2 \mathrm{~ns}) .{ }^{6}$ In contrast, along with the a

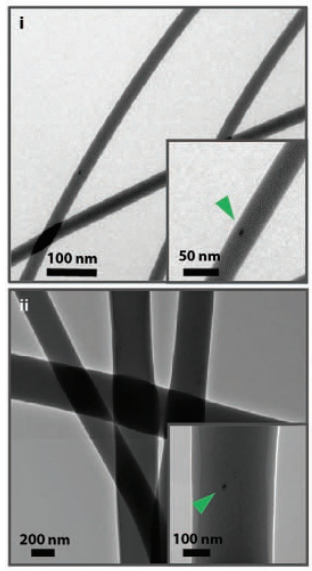

b

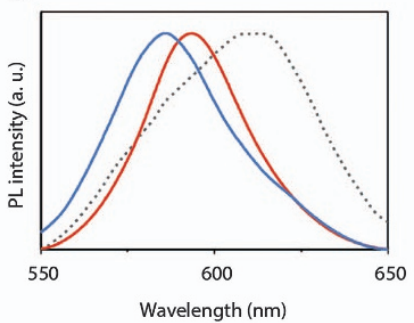

d

i) QD aggregate

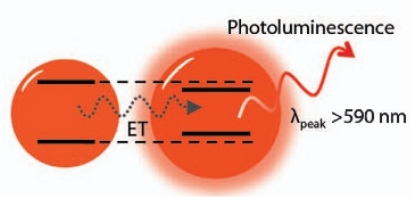

C

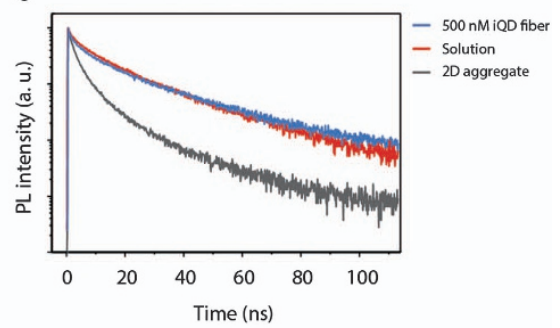

ii) Isolated QD

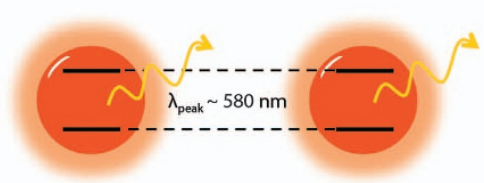

Figure 2 Monomeric features of isolated quantum dots (iQDs) in the nanofiber. (a) Transmission electron microscope (TEM) images of iQDs (green arrows) in the fiber with diameters of (i) $\sim 25 \mathrm{~nm}$ and (ii) $\sim 250 \mathrm{~nm}$. (b and c) Steady-state photoluminescence spectra and the photoluminescence decay profiles, respectively, of $\mathrm{iQDs}$ in the nanofiber (blue), QD solution (red) and two-dimensional QD aggregates (gray). (d) Brief scheme of the energy transfer processes in (i) QD aggregates (exciton coupled) and (ii) isolated QDs. 
nearly monoexponential decay indicative of the effective suppression of energy transfer processes between iQDs (Figure 2d(ii)), iQDs in the nanofiber exhibit a similar photoluminescence spectrum and decay profile to iQDs in solution.

A single-molecule spectroscopic study was performed, as this is the most straightforward approach to directly demonstrate the monomeric properties of iQDs. For this purpose, 250-nm and 1- $\mu \mathrm{m}$ fiber samples were prepared with a 5-nM QD solution, a concentration 100 times lower than that of the previous samples used for ensemble spectroscopic measurements. Furthermore, we prepared an extremely narrow nanofiber ( $\sim 25 \mathrm{~nm}$ in diameter) with a $50 \mathrm{~nm}$ QD concentration. Real-time wide-field images show that each iQD can be discriminated, emitting light as a bright spot along the fibers with diameters of $25 \mathrm{~nm}$ (Supplementary Figure S6) and $250 \mathrm{~nm}$ (Figure 3a). Over $500 \mathrm{~s}$, each iQD was observed to exhibit blinking behavior (Figure 3a and Supplementary Video V1 in the Supplementary Information), and the signals observed in all the images were accumulated over that time window (Figure 4). These cumulative images show all the QDs in the fibers and indicate that among the fibers prepared with the same QD concentration, longer distances between QDs are observed in the narrower fibers, which is in a good agreement with the Monte-Carlo simulation (Supplementary Figures S7 and S8 and Supplementary Note 2). Surprisingly, although an extremely narrow fiber $(\sim 25 \mathrm{~nm})$ was prepared with $50 \mathrm{~nm}$ QD solution, 10 times higher than the other samples, much farther distances between QDs were observed. As shown in Figure 3b, iQDs were also observed in the nanofiber by single-molecule confocal microscopy. The photoluminescence spectrum obtained from a single QD was similar to those of iQDs measured by ensemble measurements (Figure 3c). In addition, the photoluminescence decay curve of
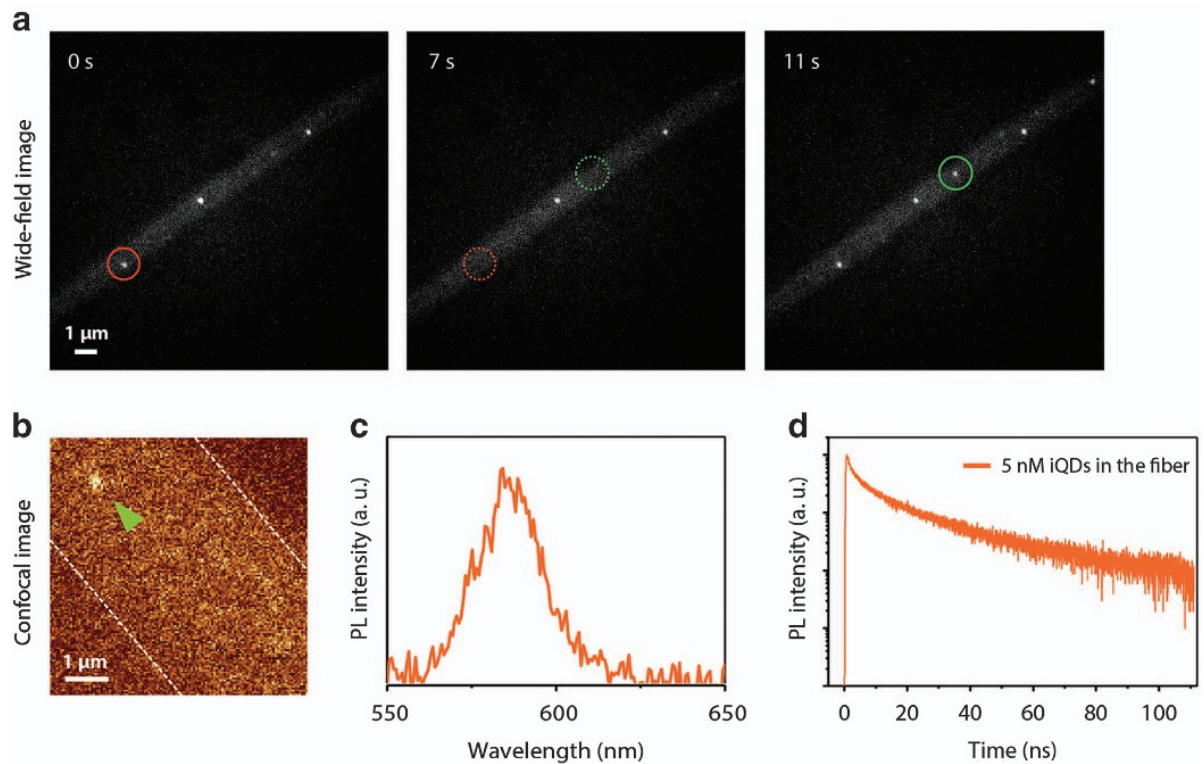

Figure 3 Single-molecule spectroscopic measurements of isolated quantum dots (iQDs) in the $250 \mathrm{~nm}$ nanofiber. (a) The blinking behavior of iQDs in the nanofiber is shown in the single-molecule wide-field images. (b) Photoluminescence scan image of an iQD in the nanofiber. (c) and (d) The photoluminescence spectrum and photoluminescence decay profiles, respectively, of a single iQD in the nanofiber (orange).
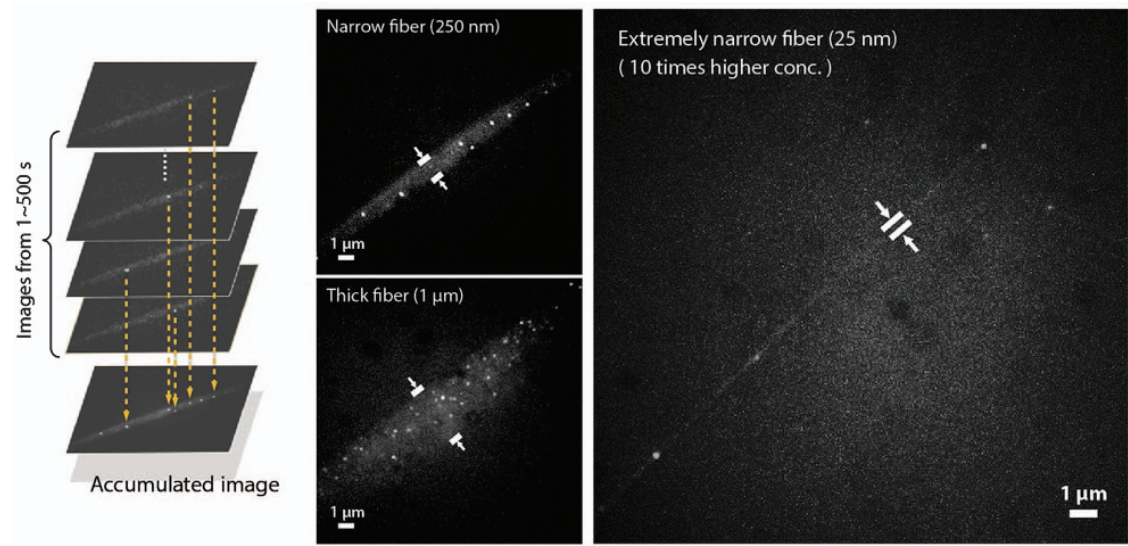

Figure 4 Images accumulated over $500 \mathrm{~s}$ show cumulative signals from all slides. Accumulated images directly show the spatial distribution of quantum dots (QDs) in the nanofiber. Although the thick and the narrow nanofiber $(\sim 1 \mu \mathrm{m}$ and $\sim 250 \mathrm{~nm}$, bottom and top in the middle, respectively) were fabricated with a $5 \mathrm{~nm}$ QD solution, the extremely narrow fiber ( $\sim 25 \mathrm{~nm}$, right) was prepared with a $50 \mathrm{~nm}$ QD solution, 10 times higher than the concentration used for the thicker fibers $(1 \mu \mathrm{m}$ and $250 \mathrm{~nm})$. 
a single QD was very close to those obtained from ensemble measurements of iQDs (Figure 3d). ${ }^{20}$ In the case of iQDs in the nanofiber, all the ensemble spectroscopic data are in agreement with the single-molecule spectroscopic data, clearly demonstrating the monomeric character of iQDs in the nanofiber.

We tested the ability of these nanofiber-embedded iQDs as a new strategy for improving the performance of dye-sensitized solar cells (DSCs) (Figure 5a). A recent study has demonstrated that the FRET concept is applicable to DSCs to fill in the absorption gaps of dye sensitizers and that this approach increases the overall light-harvesting efficiency of DSC systems, which are not able to absorb all the light. ${ }^{8,9}$ Hardin et al. ${ }^{8}$ reported an unattached energy donor (ED) dissolved in electrolyte solution, excited by higher energy photons and efficiently transferring its excitation energy to a dye sensitizer (DS), which increases the absorption spectral bandwidth of the cell and improves the power conversion efficiency from 2.55 to $3.21 \%$. However, the cell performance is decreased because of several factors: ${ }^{8-9,19,21}$ (1) dynamic quenching by electrolytes $\left(\mathrm{I}_{3}{ }^{-}\right)$, (2) self-absorption between EDs and (3) the formation of aggregates between the ED and DS. We demonstrated that iQDs encapsulated by the nanofiber can overcome the problems described above and also can efficiently transfer energy to dye sensitizers, increasing the overall power conversion efficiency and durability of the DSC.

The working electrodes of the devices were coated with anatase $\mathrm{TiO}_{2}$ nanoparticles $(15 \mathrm{~nm})$ by an electrostatic spray method in which the nanoparticles formed themselves into a hierarchically structured mesoporous bead, which serves to maximize the performance of the devices. ${ }^{22,23}$ Nanofiber-containing devices (Figure 5a(i)) were fabricated by electrospinning iQD nanofibers simultaneously with electrostatic spraying (Supplementary Figure S9 and Supplementary Video $\mathrm{V} 2$ ). As seen in SEM images (Figure $5 \mathrm{a}(\mathrm{ii})$ ), this process formed mesoporous $\mathrm{TiO}_{2}$ beads entangled with nanofibers $(5-15 \mathrm{~nm})$; the extreme narrowness of the nanofiber was achieved by decreasing the humidity and the concentration of the polymer. This state-of-the-art method was effective in increasing the contacts between $\mathrm{TiO}_{2}$ nanoparticles and nanofibers and especially in positioning the iQDs (as EDs) in close proximity ( $<10 \mathrm{~nm}$ ) to the DS to ensure efficient energy transfer. ${ }^{19,24}$ The zinc porphyrin dye ( $\beta$-(4-ethynylbenzoic acid)-substituted zinc porphyrin, ZnEP1; Figure 5a) recently reported by our lab was chosen as the DS of the DSCs to obtain a power conversion efficiency of $5.9 \% .{ }^{25}$ Figure $5 \mathrm{~b}$ shows two routes for charge separation in this device. ZnEP1 anchored on the $\mathrm{TiO}_{2}$ nanoparticle absorbs 3 and $2.2 \mathrm{eV}$ photons of the Soret and Q-bands (Supplementary Figure S10), respectively, and then transfers an electron into the $\mathrm{TiO}_{2}$ nanoparticle and a hole into the electrolyte. At the same time, an iQD in the nanofiber strongly absorbs the light with various energies $(>2.2 \mathrm{eV})$, and its efficient energy transfer to ZnEP1 can be achieved with a spectral overlap $(\sim 2.2 \mathrm{eV})$ between the emission spectrum of iQD and the absorption spectrum (Q-bands) of ZnEP1, stimulating charge separation of ZnEP1.

The excitation transfer efficiency is the probability of energy transfer from iQD to ZnEP1. The excitation transfer efficiency for an iQD is dependent on the rate of Förster energy transfer at a certain position $\vec{x}, k_{\mathrm{FRET}}(\vec{x})$, relative to the overall decay rate including the natural a

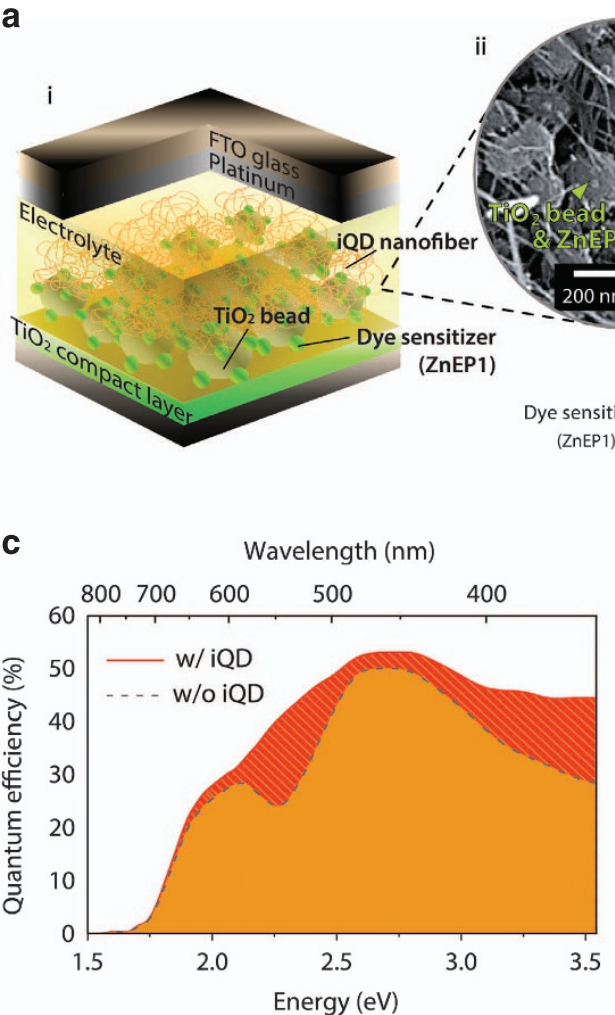

b

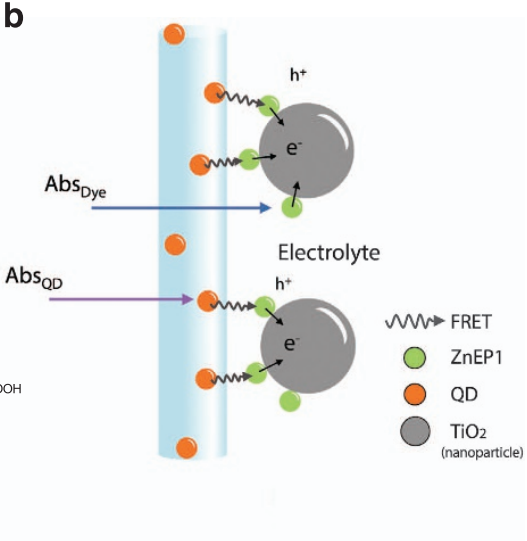

d

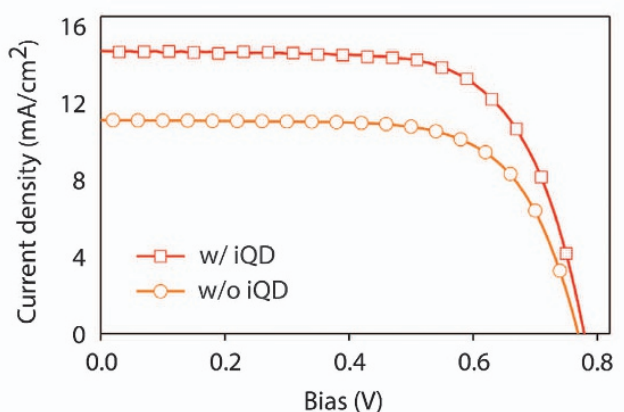

Figure 5 Structure and characterization of a light-harvesting device using isolated quantum dots (iQDs) as an auxiliary light-harvester. (a) (i) Schematic device structure and (ii) SEM image of mesoporous beads composed of $\mathrm{TiO}_{2}$ nanoparticle $(15 \mathrm{~nm})$ aggregates entangled with iQD nanofibers (5-15 nm). (b) Light-harvesting mechanism of this device. A detailed schematic representation of the film morphology is presented in Supplementary Figure S9. (c) External quantum efficiency versus photon energies of the device with and without iQDs (red and orange, respectively). (d) Photocurrent density-voltage $(J-V$ curve of the device with and without iQDs (red and orange, respectively). 
Table 1 Photovoltaic (PV) characteristics

\begin{tabular}{lccc}
\hline & With 500 nm iQDs & Without iQDs & Change (\%) \\
\hline $\mathrm{J}_{\mathrm{SC}}\left(\mathrm{mAcm}^{-3}\right)$ & 14.72 & 11.10 & 32 \\
$\mathrm{~V}_{\mathrm{OC}}(\mathrm{mV})$ & 779 & 770 & 1.17 \\
Fill factor & 0.65 & 0.69 & -5.80 \\
PCE (\%) & 7.4 & 5.9 & 25.4 \\
\hline
\end{tabular}

Abbreviations: iQDs, isolated quantum dots; $J_{\mathrm{SC}}$ : short-circuit current density; $V_{\mathrm{OC}}$, open-circuit voltage; PCE: power conversion efficiency.

The uncertainty of the photovoltaic parameters is estimated to be $3 \%$.

decay rate of $\mathrm{iQD}\left(k_{0}\right)$ and the quenching rate $\left(k_{\mathrm{Quench}}\right):{ }^{8}$

$$
\operatorname{ETE}(\vec{x})=\frac{k_{F R E T}(\vec{x})}{k_{0}+k_{\text {Quench }}+k_{F R E T}(\vec{x})}
$$

In this system, the incorporation of iQDs encapsulated by the nanofiber can maximally suppress possible quenching processes by (1) nearby QDs and (2) electrolytes $\left(k_{\text {Quench }} \sim 0\right)$. As shown in the above spectroscopic data (Figure 2c), this technique allows for the reliable isolation of QDs with a sufficient distance and effective attenuation of the energy transfer among QDs, termed self-absorption; dynamic quenching by electrolytes is also minimized because of the polymer fiber structure enveloping iQDs. If EDs are dissolved in electrolyte solution, ${ }^{8}$ however, energy transfer from ED to DS should be decreased by the above two interactions. ${ }^{21}$ In equation (1), $k_{\mathrm{FRET}}(\vec{x})$ is a function of the separation distance between an $\mathrm{QQD}$ and nearby ZnEP1, given by $k_{\mathrm{FRET}}=k_{0} \cdot\left(R_{\mathrm{o}}\right)^{6} / r^{6}$, where $r$ is the separation distance. ${ }^{26,27}$ As they are embedded in the extremely narrow fiber, many iQDs are expected to have separation distances $(<10 \mathrm{~nm})$ within the effective FRET region, ${ }^{19,24}$ and consequently a rapid FRET rate is expected (high $k_{\text {FRET }}$ ). Moreover, as the natural photoluminescence decay rate of $\mathrm{QD}, k_{0}$, is extremely slow $\left(\sim(24 \mathrm{~ns})^{-1}\right)$ relative to $k_{\mathrm{FRET}}\left(k_{0}<<k_{\mathrm{FRET}}\right)$, iQDs in close proximity to ZnEP1 are expected to have an excitation transfer efficiency value of near unity.

Figure $5 c$ presents the external quantum efficiency of DSCs to the photon energy with and without iQDs. An increase in the external quantum efficiency of DSCs with iQDs in the $2.2-3.5 \mathrm{eV}$ region compared with the control device without iQDs was attributed to the efficient energy transfer from iQDs to ZnEP1. Figure $5 \mathrm{~d}$ shows the photocurrent density-voltage $(J-V)$ characteristics of DSCs with and without $\mathrm{QQDs}$ in the nanofiber under AM $1.5 \mathrm{G}$ conditions. DSCs without iQDs exhibited a power conversion efficiency of 5.9\%, and those with $500 \mathrm{~nm}$ iQDs in the nanofiber exhibited a power conversion efficiency value of $7.4 \%$. Although $\mathrm{ZnEP} 1$ has a relatively low absorption coefficient $\left(\sim 1.5 \times 10^{4} \mathrm{M}^{-1} \mathrm{~cm}^{-1}\right)$ in the spectral overlap region $^{25}$ with the emission of iQD $(2.2 \mathrm{eV})$, the DSCs containing iQDs exhibited a significant increase in power conversion efficiency (25.4\%); however, if a dye sensitizer with large absorption coefficient in the spectral overlap region is incorporated into the system, much better device performance will be possible. This improvement in power conversion efficiency is attributed to the increase in shortcircuit photocurrent density $\left(J_{S C}\right)$ caused by an increase in the external quantum efficiency from $2 \mathrm{eV}$ as shown in Figure $5 \mathrm{c}$ (see also Table 1). In previous methods, EDs dissolved in electrolyte solution often suffer from several interactions, which changes their electronic character and finally causes the EDs to quench in the excited state before electron transfer can occur. ${ }^{9}$ Moreover, iQDs encapsulated by the nanofiber can attenuate the effects of interactions such as dynamic quenching (iQD-electrolyte), ${ }^{19,21}$ self-absorption (among iQDs) ${ }^{4-7}$ and aggregate formation (iQD-ZnEP1), leading to an increase in the absorption of the cell, its durability and, consequently, the overall light-harvesting performance of the device.

\section{CONCLUSION}

We have computationally and experimentally demonstrated that the spatial distribution of QDs can be controlled by manipulating the diameter of the nanofiber encapsulating QDs. The distance between adjacent dots tuned by the radius of the nanofiber was directly observed by single-molecule wide-field spectroscopy, and these results were in agreement with Monte-Carlo simulations. Using this reliable isolation technique, we can obtain a thick iQD nanofiber film that exhibits sufficient photoluminescence either for the ensemble spectroscopic study of iQDs or for application to light-harvesting devices. The photoluminescence spectra as well as decay curves clearly demonstrate the absence of energy transfer from higher to lower band gap QDs. Moreover, the spectral similarity of single iQDs to ensemble spectroscopic data strongly suggests that the iQDs are monomeric, without inter-dot interactions. These characteristics are clearly distinct compared with a closely-packed film or even a solution. We have successfully improved the performance of dye-sensitized solar cells from 5.9 to $7.4 \%$ due to the efficient energy transfer from iQDs to dye sensitizer, with the confinement of QDs to nanofibers preventing the problematic interactions of QDs.

To the best of our knowledge, this work is the first comprehensive investigation of the isolation of QDs in a thin film and light-harvesting devices with one-dimensional spatial confinement as well as their spectroscopic characterization on both the single molecule and ensemble levels. This study provides a general strategy that could potentially be useful for the spatial control of other functional moieties in solid-state devices as well as the fundamental study of the ensemble features of monomeric functional moieties.

\section{CONFLICT OF INTEREST}

The authors declare no conflict of interest.

\section{ACKNOWLEDGEMENTS}

This work was supported by the Global Frontier R\&D Program of the Center for Multiscale Energy Systems funded by the National Research Foundation under the Ministry of Science, ICT \& Future, Korea (2012-8-2081; DK). The authors gratefully acknowledge the support from the KIST Institutional Programs (Project 2E25371) (DYK).

Author contributions: YJC and DH conducted the fabrication and measurements. YJC, DH and DYK conceived the idea. DK supervised the study. YJC, HC and DK wrote the manuscript. All authors contributed to data analysis and reviewed the manuscript.

1 lengo, E., Zangrando, E., Minatel, R. \& Alessio, E. Metallacycles of porphyrins as building blocks in the construction of higher order assemblies through axial coordination of bridging ligands: solution- and solid-state characterization of molecular sandwiches and molecular wires. J. Am. Chem. Soc. 124, 1003-1013 (2002).

2 Geim, A. K. Graphene: status and prospects. Science 324, 1530-1534 (2009).

3 Wang, F., Zhang, Y., Tian, C., Girit, C., Zettl, A., Crommie, M. \& Shen, Y. R. Gatevariable optical transitions in graphene. Science 320, 206-209 (2008).

4 Koole, R., Liljeroth, P., de Mello Donegá, C., Vanmaekelbergh, D. \& Meijerink, A. Electronic coupling and exciton energy transfer in CdTe quantum-dot molecules. J. Am. Chem. Soc. 128, 10436-10441 (2006).

5 Mićić, O. I., Jones, K. M., Cahill, A. \& Nozik, A. J. Optical, electronic, and structural properties of uncoupled and close-packed arrays of InP quantum dots. J. Phys. Chem. B 102, 9791-9796 (1998).

6 Achermann, M., Petruska, M. A., Crooker, S. A. \& Klimov, V. I. Picosecond energy transfer in quantum dot Langmuir-Blodgett nanoassemblies. J. Phys. Chem. B 107, 13782-13787 (2003) 
7 Kagan, C. R., Murray, C. B. \& Bawendi, M. G. Long-range resonance transfer of electronic excitations in close-packed CdSe quantum-dot solids. Phys. Rev. B 54 8633-8643 (1996).

8 Hardin, B. E., Hoke, E. T., Armstrong, P. B., Yum, J.-H., Comte, P., Torres, T., Fréchet, J. M. J., Nazeeruddin, M. K., Grätzel, M. \& McGehee, M. D. Increased light harvesting in dye-sensitized solar cells with energy relay dyes. Nat. Photonics 3 , 406-411 (2009).

9 Hardin, B. E., Snaith, H. J. \& McGehee, M. D. The renaissance of dye-sensitized solar cells. Nat. Photonics 6, 162-169 (2012).

10 Yuan, W. Z., Lu, P., Chen, S., Lam, J. W. Y., Wang, Z., Liu, Y., Kwok, H. S., Ma, Y. \& Tang, B. Z. Changing the behavior of chromophores from aggregation-caused quenching to aggregation-induced emission: development of highly efficient light emitters in the solid state. Adv. Mater. 22, 2159-2163 (2010)

11 Yan, X., Cui, X. \& Li, L. Synthesis of large, stable colloidal graphene quantum dots with tunable size. J. Am. Chem. Soc. 132, 5944-5945 (2010).

12 Yan, X., Cui, X., Li, B. \& Li, L. Large, solution-processable graphene quantum dots as light absorbers for photovoltaics. Nano Lett. 10, 1869-1873 (2010).

13 Sun, L., Choi, J. J., Stachnik, D., Bartnik, A. C., Hyun, B.-R., Malliaras, G. G., Hanrath, T. \& Wise, F. W. Bright infrared quantum-dot light-emitting diodes through inter-dot spacing control. Nat. Nanotechnol. 7, 369-373 (2012).

14 Im, S. H., Kim, H.-J., Kim, S. W., Kim, S.-W. \& Seok, S. I. L. All solid state multiply layered PbS colloidal quantum-dot-sensitized photovoltaic cells. Energy Environ. Sci. 4, 4181-4186 (2011).

15 Konstantatos, G. \& Sargent, H. E. in Colloidal Quantum Dot Optoelectronics and Photovoltaics (eds Konstantatos, G. \& Sargent, H. E.) (Cambridge University Press, UK, 2013).

16 Klimov, V. I. Nanocrystal Quantum Dots 2nd edn (ed.Klimov, V. I.) (Taylor and Francis Group, Boca Raton, FL, USA, 2010).

17 Gupta, P., Elkins, C., Long, T. E. \& Wilkes, G. L. Electrospinning of linear homopolymers of poly(methyl methacrylate): exploring relationships between fiber formation, viscosity, molecular weight and concentration in a good solvent. Polymer 46, 4799-4810 (2005)

18 Ramakrishna, S., Fujihara, K., Teo, W.-E., Lim, T.-C. \& Ma, Z. An Introduction to Electrospinning and Nanofibers (ed. Ramakrishna, S.) (World Scientific Publishing Co. Pte. Ltd., To Tuck Link, Singapore, 2005).

19 Lakowicz, J. R. Principles of Fluorescence Spectroscopy 3rd edn (ed. Lakowicz, J. R.) (Springer Science+Business Media: LLC, New York, NY, USA, 2006).
20 Fisher, B. R., Eisler, H.-J., Stott, N. E. \& Bawendi, M. G. Emission intensity dependence and single-exponential behavior In single colloidal quantum dot fluorescence lifetimes. J. Phys. Chem. B 108, 143-148 (2004).

21 Margulis, G. Y., Lim, B., Hardin, B. E., Unger, E. L., Yum, J.-H., Feckl, J. M., Fattakhova-Rohlfing, D., Bein, T., Grätzel, M., Sellinger, A. \& McGehee, M. D. Highly soluble energy relay dyes for dye-sensitized solar cells. Phys. Chem. Chem. Phys. 15, 11306-11312 (2013).

22 Hwang, D., Lee, H., Jang, S.-Y., Jo, S. M., Kim, D., Seo, Y. \& Kim, D. Y. Electrospray preparation of hierarchically-structured mesoporous $\mathrm{TiO}_{2}$ spheres for use in highly efficient dye-sensitized solar cells. ACS Appl. Mater. Interfaces 3, 2719-2725 (2011).

23 Hwang, D., Kim, D. Y., Jang, S.-Y. \& Kim, D. Superior photoelectrodes for solid-state dye-sensitized solar cells using amphiphilic $\mathrm{TiO}_{2}$. J. Mater. Chem. A 1 , 1228-1238 (2013).

24 Medintz, I. L., Clapp, A. R., Mattoussi, H., Goldman, E. R., Fisher, B. \& Mauro, J. M. Self-assembled nanoscale biosensors based on quantum dot FRET donors. Nat. Mater. 2, 630-638 (2003).

25 Ishida, M., Hwang, D., Koo, Y. B., Sung, J., Kim, D. Y., Sessler, J. L. \& Kim, D. $\beta$-(Ethynylbenzoic acid)-substituted push-pull porphyrins: DSSC dyes prepared by a direct palladium-catalyzed alkynylation reaction. Chem. Commun. 49, 9164-9166 (2013).

26 Turro, N. J., Ramamurthy, V. \& Scaiano, J. C. Principles of Molecular Photochemistry: An Introduction (ed.Stiefel J.) (University Science Books, Herndon, VA, USA, 2009).

27 Shankar, K., Feng, X. \& Grimes, C. A. Enhanced harvesting of red photons in nanowire solar cells: evidence of resonance energy transfer. ACS Nano 3 788-794 (2009).

(c) (i) This work is licensed under a Creative Commons Attribution 4.0 International License. The images or other third party material in this article are included in the article's Creative Commons license, unless indicated otherwise in the credit line; if the material is not included under the Creative Commons license, users will need to obtain permission from the license holder to reproduce the material. To view a copy of this license, visit http:// creativecommons.org/licenses/by/4.0/

Supplementary Information accompanies the paper on the NPG Asia Materials website (http://www.nature.com/am) 